\title{
Rolling and Sliding of Spheres Inside Horizontal Channels
}

\author{
Ebru Demir \\ Sabanci University \\ Istanbul, Turkey \\ ebrudemir@sabanciuniv.edu
}

\author{
Serhat Yesilyurt \\ Sabanci University \\ Istanbul, Turkey \\ syesilyurt@sabanciuniv.edu
}

\begin{abstract}
Low Reynolds Number rolling and sliding motion of spheres inside cylindrical channels filled with glycerin is investigated. Experimental data are collected for channel/sphere radius ratios $\left(r_{c h} / r_{s p h}\right)$ of 1.6 and 3 , where the magnetic sphere is actuated at frequencies between 0.1-50 Hz. Magnetically actuated sphere is rotated clockwise about the $y$-axis, where the central axis of the cylindrical channel is designated as the $z$-axis and the distance between the sphere center and the channel axis is measured in $x$-direction. For $r_{c h} / r_{s p h}$ ratio of 3, we observe that the sphere translates in positive z-direction, performing "rolling". However, at smaller $r_{c h} / r_{s p h}$ ratio of 1.6, where the sphere is closely fitted inside the cylindrical channel, as the actuation frequency is increased, transition from rolling to "sliding" in the opposite direction is observed, which describes the motion of a sphere translating in negative z-direction despite its clockwise rotation about y-axis. Further increase in actuation frequency results in saturation of the lateral velocity of sphere due to stepout in both cases. Experimental results are compared to the predictions of the existing analytical models in the literature. A computational fluid dynamics (CFD) model validated against the data found in the literature is utilized to help extending the existing data and interpreting the experimental results.
\end{abstract}

Keywords—sphere, rolling, sliding, circular channel, magnetic actuation

\section{INTRODUCTION}

As our understanding of the micro- nanoscale world considerably expanded, and as the developments in the manufacturing technologies allowed us to miniaturize virtually everything, nanorobots and nanoparticles started to attract attention as an alternative means to deliver therapeutic agents to the disease inflicted sites. Targeted drug delivery not only decreases the side effects experienced due to the healthy tissues or organs being subjected to toxic agents, but it also increases the efficacy of the treatment by adjusting the dosage that needs to be administered [1]. Magnetic particles that are actuated noninvasively and that can be tracked throughout the body are promising candidates in targeted drug delivery applications. As the success of such applications depends on the accurate targeting and dosage delivery, enabling the motion of simple spherical particles in channels and understanding how those particles swim inside cylindrical conduits of different radii, such as encountered in our circulatory system, becomes crucial. Actively inducing and controlling the motion is of particular importance for the cases where the passive movement of the particle due to field gradients (e.g. chemotaxis) is not sufficient or undesirable for accurate targeting.

Various studies are conducted to understand and predict the behavior of spherical particles swimming inside conduits or near boundaries, both in the presence of a flow and in a quiescent fluid. Brenner and Happel [2] investigated the frictional drag on a sphere subjected to a Poseuille flow using method of reflections. After much iteration, they concluded that an optimum distance between the sphere center and central axis of the cylindrical conduit exists, where the drag is minimized. However, their results are valid in asymptotic cases where the distance between the sphere and the channel wall is much larger than the sphere radius. Goldman et al. [3] took near wall effects of nearly planar wall, their results can only apply when the channel radius is considerably greater than the sphere radius. Bungay and Brenner [4] studied the motion of spherical particles in a tightly fitting cylindrical conduit and proposed an improvement on the existing lubrication theories, which is still widely used in cases the sphere and the channel wall are in close proximity. Higdon and Muldowney [5] used spectral boundary element method to obtain friction coefficients of spheres swimming inside cylindrical conduits. They presented tabulated results for various $r_{c h} / r_{s p h}$ ratios, which can be used to predict swimming behavior of spheres that swim under zero net torque condition, at any distance from the channel wall. For the cases when sphere is too close to the channel wall, they employed lubrication theory. As zero torque conditions are applied, rotational friction coefficients and coupling friction coefficients are not reported.

More recently, Bhattacharya et al. [6] reported a more complete basis transformation model that overcomes the limitations of the asymptotic models. They presented translational, rotational and translation-rotation coupling friction coefficients for spheres at an arbitrary radial position, and for various $r_{c h} / r_{s p h}$ ratios. The model predicts that based on the $r_{c h} / r_{s p h}$ ratio and the distance of the sphere from the channel wall, a sphere rotating at a constant angular velocity might roll in one direction or slide in the opposite direction. Zhu et al. [7] opted for boundary element method to study the motion of spherical squirmers in capillary tubes. They focused more on the work necessary to swim, trajectory followed by 
the particles, and on the comparison of pusher and puller type swimmers. They concluded that the puller type swimmers are following a more stable trajectory, and that the presence of a confinement increases the amount of work done to achieve swimming.

Aside from these more general analyses on the motion of spheres inside confinements, more peculiar behaviors such as anomalous rolling of spheres near inclined planes are reported in the literature, namely, motion of a sphere rotating as though climbing the inclined plane despite falling downwards. One of the earliest mentions of a similar behavior is found in Cox et al.'s work [8], where the authors deduct that near the boundary, sphere should slip as it rolls based on their calculations, however, they mention that an experimental demonstration was not reported at the time. Liu et al.'s [9] work demonstrates this prediction experimentally. The authors found that, when a sphere is dropped near a planar wall, depending on the nature of the fluid used (Newtonian vs. NonNewtonian) and the angle of inclination of the wall, the sphere might perform rolling, sliding or anomalous rolling. When the wall is vertical, sphere is found to display anomalous rolling in both Newtonian and Non-Newtonian fluids, and that it shies away from the wall. They observed that the sphere transitions to normal rolling in Newtonian fluids, once the inclination of the planar wall is beyond a critical angle, however, that it still persisted in Non-Newtonian fluids. More studies reporting the behavior of the spherical particles approaching a boundary or falling near a boundary [10-14], and studies on collective behavior of multiple particles $[15,16]$ can also be found in the literature.

Despite the abundance of analytical and numerical models predicting the motion of passive spherical particles inside channels or near boundaries, experimental studies demonstrating the rolling and sliding motion of rotating swimmers are either absent or scarce, particularly for the swimming of rotating swimmers inside horizontal channels. To fill this gap and test the existing theories, an experimental study is conducted. A neodymium sphere of diameter $1 \mathrm{~mm}$ is placed inside two different glass channels of diameters $1.6 \mathrm{~mm}$ and $3 \mathrm{~mm}$, which are of length $10 \mathrm{~cm}$ and filled with glycerol. Sphere is rotated magnetically at different frequencies using two Helmholtz coil pairs and the motion of the sphere is recorded. It is shown that the motion of the sphere is affected by the ratio of the channel/sphere radii greatly. The distance between the sphere and the channel wall plays a significant role on the motion and the velocity of the swimmer. Experimental results are interpreted utilizing a resistancebased model and the coefficients presented by Bhattacharya et al. [6] and a CFD model that complements their findings.

\section{METHODOLOGY}

\section{A. Experimental}

A neodymium sphere of diameter $1 \mathrm{~mm}$ and glass tubes of diameters $1.6 \mathrm{~mm}$ and $3 \mathrm{~mm}$ are used for the experiments. 10 $\mathrm{cm}$ long cylindrical glass tubes are filled with glycerol and the magnetic sphere is placed inside the channel. One tube at a time is placed horizontally inside the experimental setup consisting of three Helmholtz coil pairs connected to Maxon drivers controlled via Labview software. Two of the coil pairs

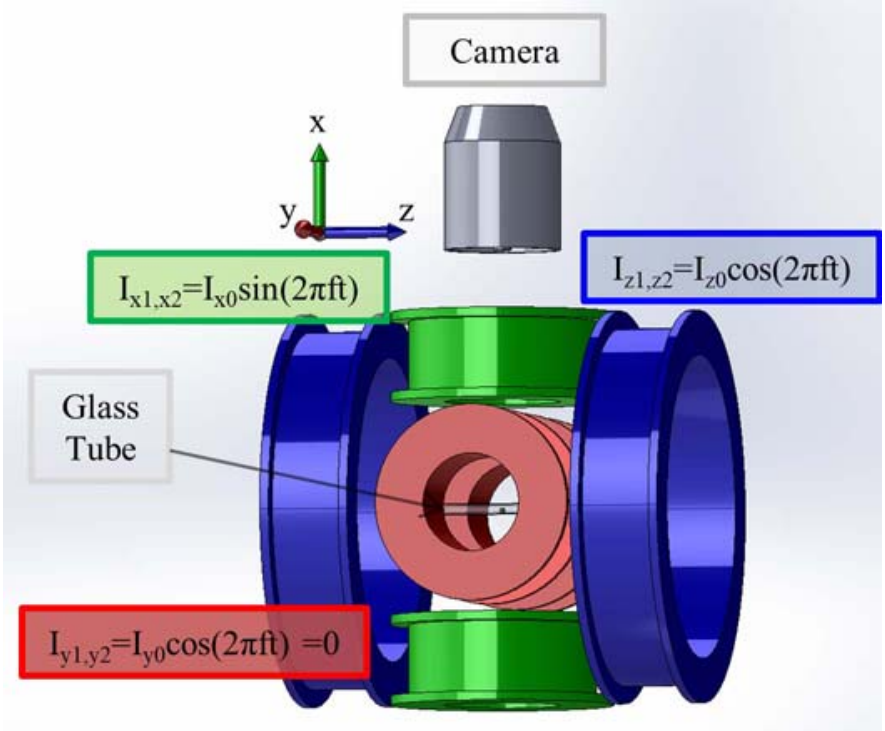

Fig. 1 Experimental setup: Magnetic field rotating about y-axis is applied using $\mathrm{x}$ (green) and $\mathrm{z}$ (blue) coil pairs. A digital microscope is used as camera to record the experiments.

( $\mathrm{x}$ and $\mathrm{z}$ pairs) are activated with sinusoidal out-of-phase currents to create a magnetic field rotating about the y-axis whereas the axis of the cylindrical channel is placed along the $z$-direction. The distance between the centers of the sphere and the axis of the cylindrical channel is measured along the $\mathrm{x}$ axis. The magnetic sphere is actuated at different magnetic field rotation rates ranging between $[0.1-50 \mathrm{~Hz}]$ and its motion is recorded using a digital microscope from above to observe the movement in the $\mathrm{x}$-direction and response to the magnetic field.

As a rotating magnetic field is applied to the magnetic sphere, its magnetic dipole moment tries to align with the direction of the applied magnetic field, which causes the rotation of the sphere. When the magnetic torque acting on the sphere due to its rotation exceeds the viscous torque acting on it due to the presence of a fluid, the sphere starts to translate along $\mathrm{z}$-axis. The relationship between the magnetic dipole moment, $\mathbf{m}$, the magnetic field, $\mathbf{B}$, and the magnetic torque, $\boldsymbol{\tau}_{\mathbf{m}}$ is given by the following equation:

$$
\boldsymbol{\tau}_{\mathbf{m}}=\mathbf{m} \times \mathbf{B}=\mu_{0} \mathbf{m} \times \mathbf{H}
$$

where $\mu_{0}$ denotes the permeability of the free space and $\mathbf{H}$ is the magnetic field strength. As implied by the cross-product, actual magnetic torque acting on the sphere at any given instance depends on the sinus of the angle between the magnetic dipole moment vector of the sphere and the magnetic field vector, and the angle depends on the net torque acting on the sphere, taking both viscous and magnetic torques into account.

Positions of the spheres are obtained through image processing via a MATLAB code, using the images recorded by the digital 
microscope directed towards yz-plane. The image processing code extracts each frame of the recording and masks the area where the channel is located. Then the background is cancelled through use of filters, such that only the image of the sphere remains as a black circle against a white background. Afterwards, the code calculates the centroid of the sphere and tracks its position. Using the position data, lateral velocity of the sphere is calculated.

\section{B. $C F D$}

CFD model used in the validation of the data extrapolation based on the data presented in Bhattacharya et al.'s study [6] solves incompressible Stokes equations, which are given as follows in non-dimensional form:

$$
-\nabla p+\frac{1}{R e} \nabla^{2} \mathbf{u}=0 \quad \text { and } \quad \nabla \cdot \mathbf{u}=0
$$

where $\mathbf{u}$ and $p$ denote the velocity vector and pressure respectively. The Reynolds number, $R e$, is based on the rotation frequency, $f$, and a length scale, $\ell$, such as the diameter of the channel: i.e. $R e=\rho \ell^{2} f / \mu$, where $\rho$ is the density and $\mu$ is the viscosity of glycerine.

No-slip boundary conditions are used on the channel wall and on the sphere surface moving with the velocity:

$$
\mathbf{u}=\mathbf{U}+\boldsymbol{\omega} \times\left(\mathbf{r}-\mathbf{r}_{\mathbf{0}}\right), \quad \mathbf{r} \in S
$$

where $\mathbf{U}$ is the swimming velocity, $\boldsymbol{\omega}$ is the angular velocity, $\mathbf{r}$ is the position, $\mathbf{r}_{0}$ is the position of the centroid, and $S$ represents the surface of the body. Force-free swimming condition is applied to the sphere surface as follows:

$$
\mathbf{F}_{\text {net }}=\int_{S} \boldsymbol{\sigma} \cdot \mathbf{n} d A=0
$$

where $\boldsymbol{\sigma}$ is the stress tensor and $\mathbf{n}$ is the local surface normal.

COMSOL Multiphysics [17] software is used to solve incompressible Stokes equations with finite element method. $\mathrm{P} 1+\mathrm{P} 1$ discretization of the fluids and MUMPS direct solver is employed for the simulations. Triangular surface mesh is applied to the sphere surface and tetrahedral elements are used to mesh all domains. Smaller mesh elements are used in the region where sphere gets close to the channel to capture the more complex dynamics in this region. Average degrees of freedom (DOF) for the results reported in this paper is $500 \mathrm{~K}$ and mesh convergence of the results are observed.

Here, it should be noted that the CFD study is conducted only to interpret our experimental results extending and using the available data presented by Bhattacharya et al [6]. Since their study does not report on the case where $r_{c h} / r_{s p h}=1.6$, a direct comparison of the experimental results with the theoretical model is not possible. However, data presented for $r_{c h} / r_{s p h}=2$ and $r_{c h} / r_{s p h}=3$ are sufficient to demonstrate the effect of $r_{c h} / r_{s p h}$ ratio on thesphere's behavior.

\section{RESULTS AND DISCUSSION}

Fig. 2 depicts the velocities of the neodymium sphere of diameter $1 \mathrm{~mm}$, swimming inside cylindrical channels of diameters $1.6 \mathrm{~mm}$ (Fig. 2/a) and $3 \mathrm{~mm}$ (Fig. 2/b) at different actuation frequencies.

The influence of the channel-to-sphere radius ratio, $r_{c h} /$ $r_{s p h}$ on the swimming behavior of the sphere is easily discernible from the significantly different velocity profiles obtained for the same sphere (Fig. 2). In the channel with 3 $\mathrm{mm}$ diameter, the sphere goes through two phases, namely, the rolling and step-out (Fig. 2/b). Rolling motion of the sphere implies lateral movement of the sphere in the positive $\mathrm{z}$ direction, while it rotates clockwise in the y-direction. The term step-out indicates that the sphere cannot sustain a synchronous rotation with the magnetic field. In the rolling phase, the velocity demonstrates a linear dependence on the rotation frequency. At frequencies greater than $14 \mathrm{~Hz}$, step-out behavior is observed. First the sphere starts to slow down, as the rotation of the sphere loses sync with the rotation of the magnetic field. Velocity starts to decrease until it saturates at higher actuation frequencies.

In the narrow channel, sphere displays a more complex behavior, where the velocity exhibits three different phases with increasing frequencies. At rotation frequencies ranging between $0.1-0.3 \mathrm{~Hz}$, the sphere rolls in the positive $\mathrm{z}$-direction. However, as the frequency increases up to $10 \mathrm{~Hz}$, the sphere starts to swim in negative $z$-direction despite its clockwise rotation about y-axis. This behavior will be referred to as "sliding" in the remainder of this paper. Even though the sphere's movement transitions from rolling to sliding, velocity displays an almost linear dependency on frequencies between $0.1-10 \mathrm{~Hz}$. After $10 \mathrm{~Hz}$, the behavior can be attributed to stepout.

The motion of the sphere inside the narrow channel can be explained as follows: At very low frequencies, sphere is in contact with the channel wall, and the magnetic field is rotating so slowly, that the sphere's magnetic dipole moment can sync with it easily. In this phase, the balance between the magnetic torque and friction torque on the sphere result in synchronous rolling of the sphere in the z-direction. However, as the sphere rotates faster, it loses its traction and due to the small diameter of the channel, a pressure difference in the front and at the wake of the sphere start builds up (Fig. 2/a), effectively pushing the sphere in the negative z-direction [18]. Hence the sphere slows down with increasing frequency. As the pressure difference between the two sides of the sphere increases, it starts to slide in the opposite direction. Increasing the actuation frequency rotates the sphere faster, which increases the pressure difference, thus the sphere accelerates in negative z-direction. Change in the distance of the sphere from the channel wall can also contribute to this behavior and explain slight deviations from the linear profile. As the actuation frequency gets higher, the sphere might achieve liftoff from the channel wall [19-22], and become more susceptible to the pressure difference, however, such minor changes in the distance between the sphere and the channel 
wall cannot be quantified experimentally. When the actuation frequency exceeds $10 \mathrm{~Hz}$, sphere starts to become out of sync with the rotating field, and its sliding motion is slowed down until a saturation velocity is reached. Another remark that must be made here is that the saturation velocity is negative for the sphere in the tighter confinement, whereas it was positive for the sphere swimming in the wide channel.

Bhattacharya et al.'s [6] theoretical findings confirm our interpretation of the experimental data presented. Firstly, their model also captures the effect of $r_{c h} / r_{s p h}$ ratio on the movement of the spherical particles in cylindrical confinements. They derived the translational and rotational resistance coefficients of spheres swimming inside circular channels, and reported different results for various $r_{c h} / r_{s p h}$ ratios. A closer inspection of the data presented in Bhattacharya et al. [6] shows that the transition from rolling to sliding is highly affected by the relative radii of the sphere and the channel, as well as by the distance of the spherical particle from the wall. The relationship between the friction coefficients, forces and torques acting on the sphere, and velocity and the angular velocity are given is follows [6]:

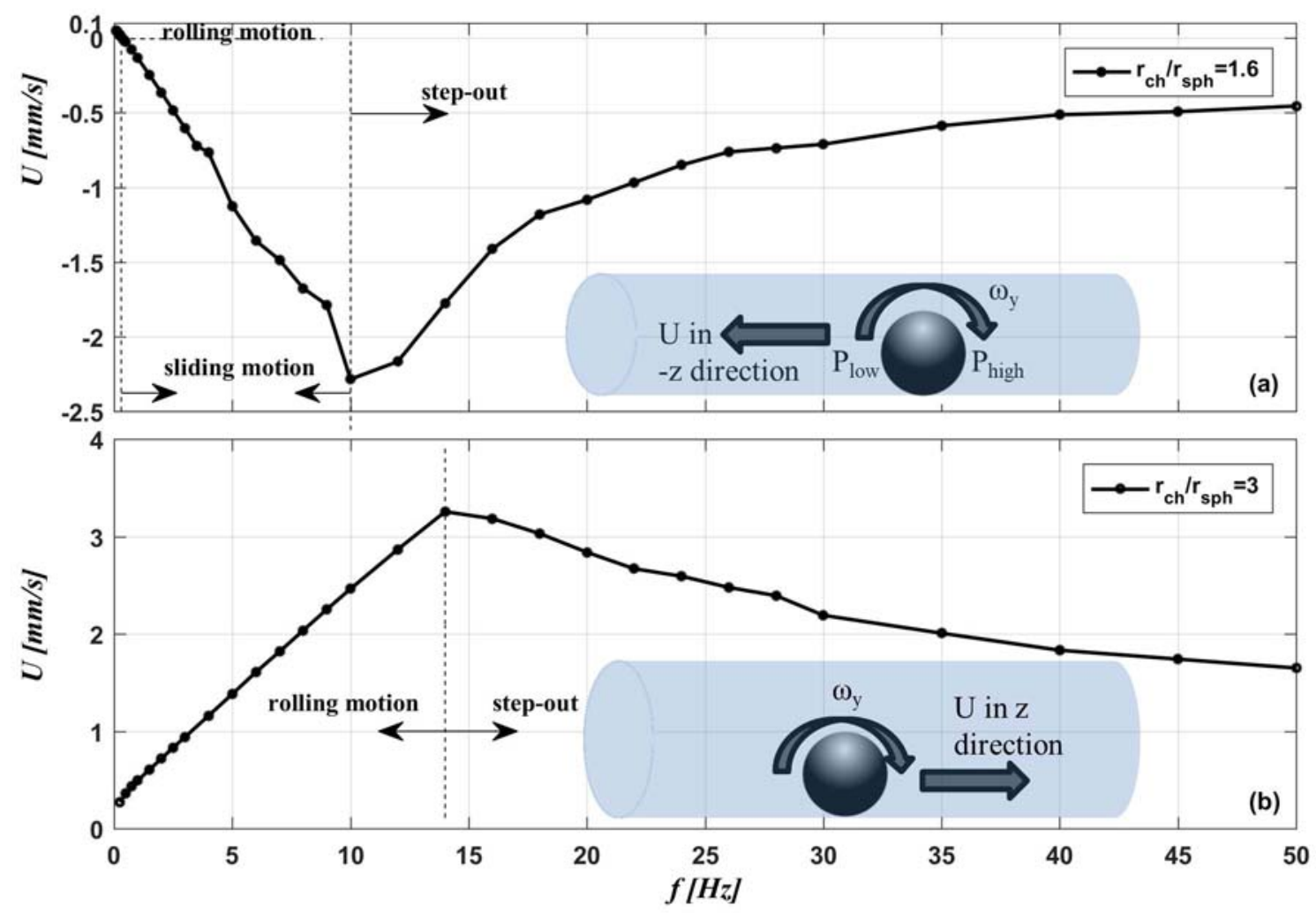

Fig. 2 Translation velocity, $U$, vs. the actuation frequency, $f$, of the sphere of diameter $1 \mathrm{~mm}$ inside cylindrical channels of (a) $1.6 \mathrm{~mm}$, and (b) 3 mm

$$
\begin{aligned}
& F^{\mathrm{tt}} \cdot \mathbf{u}+\mathbf{F}^{\mathrm{tr}} \cdot \omega+\mathbf{f}^{\mathrm{p}}+\mathbf{f}^{\mathrm{ex}}=0 \\
& \mathbf{F}^{\mathrm{rt}} \cdot \mathbf{u}+\mathbf{F}^{\mathrm{rr}} \cdot \omega+\tau^{\mathrm{p}}+\tau^{\mathrm{ex}}=0
\end{aligned}
$$

where $\mathbf{F}^{\mathrm{tt}}, \mathbf{F}^{\mathrm{rr}}, \mathbf{F}^{\mathrm{tr}}$ denote the friction coefficient tensors for translation, rotation and translation-rotation coupling respectively. $\mathbf{F}^{\mathbf{r t}}$ is the transpose of $\mathbf{F}^{\mathbf{t r}}$, whereas $\mathbf{u}$ and $\boldsymbol{\omega}$ are the translational and angular velocity vectors. Vectors $\mathbf{f}$ and $\boldsymbol{\tau}$ denote force and torque, whereas the superscripts ${ }^{p}$ and $e x$ indicate that the forces or torques occur due to the presence of a parabolic flow or an external input. Applying zero net force condition and necessary coordinate transformations, and considering that angular velocity is nonzero only about the yaxis, these equations simply reduce to:

$$
\frac{G^{\prime}}{F_{z z}^{t t}}=\frac{U}{\omega_{y}} \text { or } U=\left(2 \pi \frac{G^{\prime}}{F_{z z}^{t t}}\right) f
$$

where $G^{\prime}$ is the component of translation-rotation coupling friction coefficient related to rotation about y-axis, $U$ is the translation velocity of the sphere, $F_{z z}^{t t}$ is the z-component of the translational friction coefficient, $f$ is the rotation frequency, and $\omega_{\mathrm{y}}$ is the angular velocity about $\mathrm{y}$-axis.

Using equation (4), velocity profile of the sphere in the linear regime can be obtained. To validate our model, we focus on the cases where the sphere is almost in contact with the wall in order to observe the effect of the change in particlechannel wall distance on the velocity clearly. Since Bhattacharya et al. [6] present data for $r_{c h} / r_{s p h}=2,3$ and 4, and our ratios are $r_{c h} / r_{s p h}=1.6$ and 3, we will use the data for the ratios $r_{c h} / r_{s p h}=2$ and $r_{c h} / r_{s p h}=3$. We focus on the linear regime observed in both cases, where $r_{c h} / r_{s p h}=2$ and $r_{c h} / r_{s p h}$ $=3$. To this end, we need to find the friction coefficients $F_{z z}{ }_{z z}$ and $G$ ' near the channel wall. If $R$ is the distance between the sphere center and the central axis of the cylinder measured 
along $\mathrm{x}$-axis, distance of the sphere from the wall can be quantified with the normalized variable $\beta$ as follows:

$$
\beta=\frac{R}{r_{c h}-r_{s p h}}
$$

Bhattacharya et al. [6] report $F_{z z}^{t t}$ and $G$ ' values for a range of $\beta$ values. However, since the sphere is either in contact or near-contact with the channel wall, we needed to obtain $F_{z z}^{t t}$ and $G^{\prime}$ values for $\beta$ much closer to unity. To predict $F_{z z}^{t t}$ and $G$ ' values for greater $\beta$, we first develop a simple CFD model of the experiment using commercially available software COMSOL $^{\circledR}$ [17]. Using this model, we obtain $F_{z z}^{t t}$ values for the cases $r_{c h} / r_{s p h}=2$ and $r_{c h} / r_{s p h}=3$ to be able to compare our results directly to the reported values. Black line in Fig. 3 depicts Bhattacharya's [6] results, whereas the blue line shows the CFD results. Reasonably well agreement is achieved with Bhattacharya et al.'s results, as the maximum relative error is found to be less than $4.5 \%$ for the available data.

After this validation, we extend our results to higher $\beta$ values. Finally, we found a polynomial fit to Bhattacharya's [6] data that would agree with CFD results at $\beta$ values very
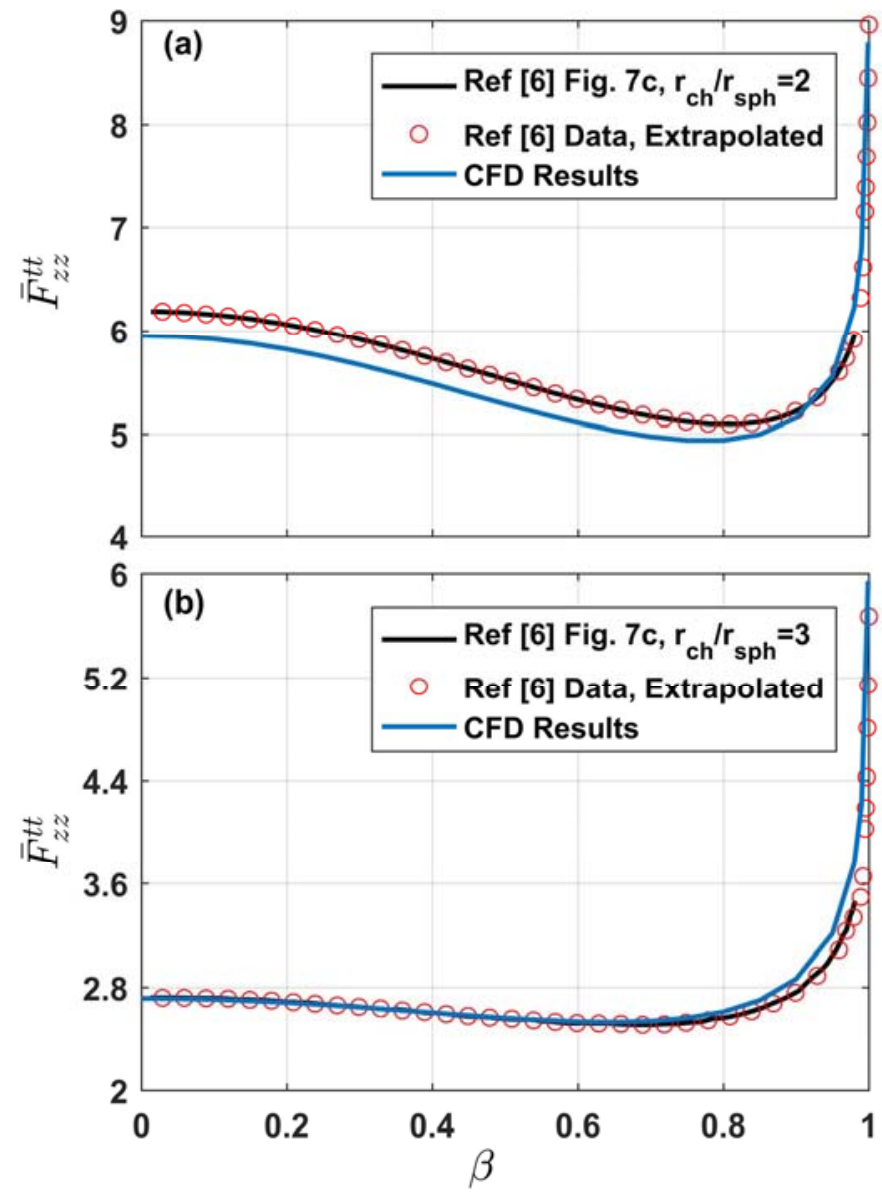

Fig. 3 Comparison of friction coefficient $F_{z z}^{t t}$ vs. $\beta$ reported by Bhattacharya et al., extrapolation of their data and data obtained from our CFD model, for $r_{c h} / r_{s p h}=2$ (a) and $r_{c h} / r_{s p h}=3$ (b). close to unity. Using the equation of the polynomial, we deduce $F_{z z}^{t t}$ and $G$ ' values for a sphere, which swims very close to the channel wall, and obtain the slope of the $U$ vs. $f$ graph in the linear region. A comparison of the data obtained from the CFD and from the extrapolation Bhattacharya's [6] results is presented in Table 1.

Table 1 exhibits the role of distance on the velocity. Even slight changes in the distance can be very effective in changing the swimming characteristics [8]. For instance, CFD model predicts that the sphere is rolling when in contact with the channel wall, but that it is sliding when it is even a fraction away from the wall when $r_{c h} / r_{s p h}=2$. The sphere accelerates in negative z-direction according to both models. The sensitivity of the velocity to the distance from the wall is clearly depicted.

Analytical results predict the sliding of the sphere against the rolling velocity, but since we use extrapolated data, the change in the sign of the velocity as the sphere approaches near contact with the wall could not be captured. CFD model on the other hand predicts the transition from rolling to sliding. For the CFD model, $\beta=0.99999$ case is practically equivalent to contact mode. Distinguishing the full contact mode in CFD from $\beta=0.99999$ configuration would require a very fine mesh and high computational power. Therefore, at $\beta=$ 0.99999 CFD model predicts the results for the contact case. However, an analytical model can distinguish between nearcontact and contact modes; but still, predictions will be within an error margin. For the wide channel, both models predicted positive velocities that increase as the sphere gets closer to the wall.

TABLE I. 2ח $G^{\prime} / F^{r T}$ Zz VALUES OBTAINED FROM EXTRAPOLATION OF THE AVAILABLE DATA AND CFD

\begin{tabular}{cccc}
\hline $\boldsymbol{R}_{\boldsymbol{c h}} / \boldsymbol{R}_{\text {sph }}$ & $\boldsymbol{\beta}$ & Bhattacharya et al. [6] & $\boldsymbol{C F D}$ \\
\hline \multirow{3}{*}{2} & 0.99 & -0.11215 & -0.16637 \\
& 0.999 & -0.026969 & -0.12085 \\
& 0.9999 & -0.018632 & -0.07139 \\
& 0.99999 & -0.017824 & 0.30988 \\
\hline \multirow{3}{*}{3} & 0.99 & 0.039709 & 0.0095974 \\
& 0.999 & 0.131 & 0.059408 \\
& 0.9999 & 0.14223 & 0.10311 \\
& 0.99999 & 0.1433 & 0.16534 \\
\hline
\end{tabular}

\section{CONCLUSIONS AND FUTURE WORK}

Motion of a sphere swimming inside horizontal cylindrical channels is investigated experimentally and computationally. It is shown that the particular dynamics of the sphere rotating and translating inside a viscous fluid is highly dependent on $r_{c h} / r_{s p h}$ and instantaneous distance of the sphere from the channel wall. Results and the model constructed to interpret them are in agreement with the analytical results found in the literature. 
As future work, relationship between the translational velocity profile of the sphere and its distance from the channel wall should be investigated. Complex resistance coefficient matrices that would relate multiple forces acting on the rotating and translating sphere to its swimming behavior should be driven. Pressure drop across the sphere due to this coupled motion needs to be quantified. Scaling down the experimental setup or conducting a nondimensional analysis is necessary for accurate prediction of the spherical particles in nanofluid solutions. 3D manipulation of the sphere trajectory is also a valuable challenge.

\section{REFERENCES}

[1] Martel, S. (2015). Magnetic nanoparticles in medical nanorobotics. Journal of Nanoparticle Research, 17(2), 75.

[2] Brenner, H., \& Happel, J. (1958). Slow viscous flow past a sphere in a cylindrical tube. Journal of Fluid Mechanics, 4(2), 195-213.

[3] Goldman, A. J., Cox, R. G., \& Brenner, H. (1967). Slow viscous motion of a sphere parallel to a plane wall-I Motion through a quiescent fluid. Chemical engineering science, 22(4), 637-651.

[4] Bungay, P. M., \& Brenner, H. (1973). The motion of a closely-fitting sphere in a fluid-filled tube. International Journal of Multiphase Flow, 1(1), 25-56.

[5] Higdon, J. J. L., \& Muldowney, G. P. (1995). Resistance functions for spherical particles, droplets and bubbles in cylindrical tubes. Journal of Fluid Mechanics, 298, 193-210.

[6] Bhattacharya, S., Mishra, C., \& Bhattacharya, S. (2010). Analysis of general creeping motion of a sphere inside a cylinder. Journal of Fluid Mechanics, 642, 295-328.

[7] Zhu, L., Lauga, E., \& Brandt, L. (2013). Low-Reynolds-number swimming in a capillary tube. Journal of Fluid Mechanics, 726, 285311 .

[8] Goldman, A. J., Cox, R. G., \& Brenner, H. (1967). Slow viscous motion of a sphere parallel to a plane wall-II Couette flow. Chemical engineering science, 22(4), 653-660.
[9] Liu, Y. J., Nelson, J., Feng, J., \& Joseph, D. D. (1993). Anomalous rolling of spheres down an inclined plane. Journal of non-Newtonian fluid mechanics, 50(2-3), 305-329.

[10] Bico, J., Ashmore-Chakrabarty, J., McKinley, G. H., \& Stone, H. A. (2009). Rolling stones: The motion of a sphere down an inclined plane coated with a thin liquid film. Physics of fluids, 21(8), 082103.

[11] Brenner, H. (1961). The slow motion of a sphere through a viscous fluid towards a plane surface. Chemical engineering science, 16(3-4), 242251.

[12] Charru, F., Larrieu, E., Dupont, J. B., \& ZENIT, R. (2007). Motion of a particle near a rough wall in a viscous shear flow. Journal of Fluid Mechanics, 570, 431-453.

[13] Jeffrey, D. J., \& Onishi, Y. (1981). The slow motion of a cylinder next to a plane wall. The Quarterly Journal of Mechanics and Applied Mathematics, 34(2), 129-137.

[14] Adamczyk, Z., Adamczyk, M., \& Van de Ven, T. G. M. (1983). Resistance coefficient of a solid sphere approaching plane and curved boundaries. Journal of colloid and interface science, 96(1), 204-213.

[15] Navardi, S., Bhattacharya, S., \& Wu, H. (2015). Stokesian simulation of two unequal spheres in a pressure-driven creeping flow through a cylinder. Computers \& Fluids, 121, 145-163.

[16] Cichocki, B., Felderhof, B. U., Hinsen, K., Wajnryb, E., \& Blawzdziewicz, J. (1994). Friction and mobility of many spheres in Stokes flow. The Journal of chemical physics, 100(5), 3780-3790.

[17] COMSOL Multiphysics ${ }^{\circledR}$ v. 5.3. www.comsol.com. COMSOL AB, Stockholm, Sweden.

[18] Navardi, S., \& Bhattacharya, S. (2010). Axial pressure-difference between far-fields across a sphere in viscous flow bounded by a cylinder. Physics of Fluids, 22(10), 103305.

[19] Hu, H. H., \& Joseph, D. D. (1999). Lift on a sphere near a plane wall in a second-order fluid. Journal of non-newtonian fluid mechanics, $88(1)$, 173-184.

[20] Bhattacharya, S., \& Navardi, S. (2013). Radial lift on a suspended finitesized sphere due to fluid inertia for low-Reynolds-number flow through a cylinder. Journal of Fluid Mechanics, 722, 159-186.

[21] Krishnan, G. P., \& Leighton Jr, D. T. (1995). Inertial lift on a moving sphere in contact with a plane wall in a shear flow. Physics of Fluids, 7(11), 2538-2545.

[22] King, M. R., \& Leighton, Jr, D. T. (1997). Measurement of the inertial lift on a moving sphere in contact with a plane wall in a shear flow. Physics of Fluids, 9(5), 1248-1255. 